\title{
Oesophageal cancer treatment in North East Thames region, 1981: medical audit using Hospital Activity Analysis data
}

\begin{abstract}
Figures from the Hospital Activity Analysis in the North East Thames region in 1981 were used to perform a medical audit on oesophageal cancer treatment. Four hundred and forty four patients were admitted with this diagnosis; 80 had been intubated without a thoracotomy or laparotomy, and 73 had had surgery (two thirds radical and one third palliative) with an overall operative mortality of $33 \%$. Fifty five patients had had radiotherapy and 179 patients had no recorded operation or investigation. One hundred and seventy seven different consultants had looked after all these inpatients, most being general surgeons. Only five consultants had looked after 10 or more patients each year. From a calculated estimate of a total 286 patients in the region, $28 \%$ had palliative intubation and $25 \%$ had surgery; $20 \%$ of all the patients had radiotherapy either as a radical or palliative treatment, the remainder having no recorded therapeutic procedure. One hundred and eighty seven patients $(66 \%$ of the calculated total) died in hospital.
\end{abstract}

Investigation and treatment do not seem to be limited by lack of money, but money is being wasted by admitting patients for terminal care into acute hospital beds. It would be more humane for these patients to die at home or in a hospice if they wished.

\section{Introduction}

The number of deaths from oesophageal cancer in England and Wales is rising. In 1978 there were 3708 deaths, an increase of about one third more than 10 years before. ${ }^{1}$ Surgery is advised in the minority of patients because either the disease is too widespread or the patient is old and unfit. There is controversy about the mortality associated with these operations and disagreement whether radiotherapy should be advised more often, but in numerical terms most cases are incperable and receive no definitive treatment. ${ }^{2} 3$ When all patients are considered the survival rate at one year is $18 \%$ and at five years $5 \%{ }^{4}$ This review is an audit of the statistics in the North East Thames region and reports what is actually happening in hospital, how many patients have radical or palliative surgery and the mortality of these procedures, how many patients are intubated, have radiotherapy, and die in hospital with or without treatment. The figures, obtained from Hospital Activity Analysis, are not perfect, but they are the best available.

\section{Methods}

A list of all inpatient episodes with a main or secondary diagnosis of oesophageal cancer was obtained from the Hospital Activity Analysis data for the North East Thames Regional Health Authority in 1981 and also 1980. There were four teaching hospitals in the region in 1981, and 16 districts, all of which had one or more hospitals doing acute surgery. Figures for inpatient hospital procedures were obtained from

The London Hospital, London E1 1BB

RICHARD EARLAM, MCHIR, FRCS, consultant surgeon
52 different hospitals but there were 74 additional hospitals out of the total of 136 in which no patients with oesophageal cancer were looked after. Since operations were performed only in 23 out of the 52 hospitals where patients with oesophageal cancer were cared for, it must be presumed that the remainder represented convalescent, hospice, or long stay hospitals.

\section{Results}

In 1981, 444 patients were admitted to hospitals of the North East Thames region with a diagnosis of oesophageal cancer. There were 645 different admission episodes with a mean per patient of 1.4 ; 73 patients had surgery, of whom 49 had the tumour removed and an oesophagogastrectomy performed, but in 24 a palliative procedure only was done. Eighty patients had endoscopic intubation without a thoracotomy or a laparotomy; 291 had no definite therapeutic treatment, either no procedure at all (179) or only a diagnostic endoscopy (112). In 1981 or before radiotherapy was given to 55 of the 444 patients. A total of 187 died in hospital.

Radical surgery-Forty nine patients had an oesophagogastrectomy, of whom 17 died in hospital. Their average age was 64 and the mean stay in hospital was 31 days, with six before operation and 25 afterwards. Seven of the deaths in hospital occurred less than 10 days after the operation and seven over 30 days after the operation. The mean number of days after the operation when death occurred was 27.

Palliative surgery-A laparotomy or a thoracotomy was performed in 24 patients without a radical resection being possible. Seven of the 24 died in hospital. Their average age was 74 and the hospital stay 21 days (six before and 15 after operation). The mean time of death was on the 12th day. Only one gastrostomy was reported. In five of the 24 there was no record of intubation; one of these patients had a gastrostomy, the others presumably had no further palliative surgical procedure done.

Intubation only-Eighty patients (mean age 72) had the tumour intubated. This does not include the 19 intubated in the palliative surgery group; 27 of the 80 died in hospital after a mean stay of seven days. The average duration of stay in hospital for all 80 patients was 18 days ( 4.5 before and 13.5 after operation)

No therapeutic procedure-A total of 179 patients had no operative or investigative procedure recorded, and 106 died in hospital; 112 had an endoscopy done with or without biopsy, and 30 died in hospital. The mean hospital stay of this whole group of 291 patients was 24 days.

Number of days in hospital-The whole group of 444 patients spent a total of 10355 days in hospital. Given the average of 1.4 admissions per patient, the mean length of stay was 23 days $(15 \%$ of the total number of days in hospital). To break the total into groups, these were as follows: radical surgery 1502 days, palliative surgery 509 days, intubation 1432 days, and no therapeutic procedure 6912 days.

Radiotherapy-Fifty five patients had a course of radiotherapy either in 1981 or earlier. The mean age was 70 with 1.9 admissions to hospital, resulting in a total of 1429 hospital days. The patients who had radiotherapy have been included in other relevant groups.

Consultant care-Some 177 different consultants looked after the 444 patients (mean number of patients per consultant $2 \cdot 5$ ): 63 general surgeons; 58 general physicians; 12 geriatricians; 11 radiotherapists; 10 ear, nose, and throat surgeons, and nine thoracic surgeons looked after the majority. Thirty four different consultants performed 49 radical operations, general surgeons doing 37 , thoracic surgeons nine, and those in other specialties the remainder. Only five consultants looked after more than 10 patients a year; four were general surgeons and one was a physician. In 1981 no consultant cared for more than 17 patients with oesophageal cancer.

Death in hospital-A total of 187 patients died in hospital with oesophageal cancer; many of these had been in hospital before because the mean number of admissions for this group of patients was 1.5. Their average age was 72 ; the mean number of days for the last visit was 17 . 
Seventy four were in for less than one week and $\mathbf{4 5}$ for more than three weeks. Fifty one died after operation; 27 occurred after intubation but 109 of the patients dying in hospital had had no recorded therapeutic procedure in 1981.

Admissions to hospital: 1980-The Hospital Activity Analysis records for 1980 were studied to see whether the pattern for that year was similar and to find out how many of the patients admitted to hospital in 1981 had been admitted or diagnosed in the previous year. Thirty four out of the 444 patients admitted in 1981 had been in hospital for diagnosis or treatment in 1980 . In this year there were 420 admissions with 175 deaths in hospital. Fifty six had had radical surgery with 24 hospital deaths. Thirty four had had palliative surgery, 50 intubation, and 281 no definite therapeutic procedure. Fifty five had had radiotherapy recorded as a treatment.

Annual incidence of patients with oesophageal cancer in North East Thames-The population of the North East Thames region in 1981 was 3694300 , of whom 1790400 were male and 1903900 female. In 1979 (the latest available year) 229 deaths were recorded with oesophageal cancer, of which 127 occurred in men and 102 in women. ${ }^{5}$ Cancer registration in the region is incomplete and unreliable and has been suspended before reorganisation. Nevertheless, with the latest available figures, the 1981 registration for oesophageal cancer in England and Wales was $9 \cdot 3$ per 100000 for men and 6.3 per 100000 for women. If the overall registration rate for oesophageal cancer based on 1981 figures for England and Wales is applied to the North East Thames region population in 1981 and adjusted for age differences, the actual number of patients diagnosed with oesophageal cancer was estimated to be 286, of whom 166 would have been men and 120 women. Forty eight of the men and 63 of the women were calculated to be 75 or over, representing $35^{\circ} \circ$ of the total.

\section{Discussion}

This review aims at clarifying exactly what was happening to patients with oesophageal cancer in hospital. I chose the North East Thames region because that is where I work, no one special centre dictates therapy, and the population of about 3.7 million is sufficiently large for analysis. The disease is rare and this number was necessary for the study of different treatments.

A true epidemiological approach analysing all patients is impossible using the inpatient Hospital Activity Analysis because all the patients in a community need to be studied and this requires accurate figures for incidence and prevalence. In 1981 there were 645 different admissions to the hospitals of the North East Thames region, concerning 444 patients who could be identified by their hospital number.

With figures for the incidence of oesophageal cancer in England and Wales applied, age adjusted, to the population of the North East Thames region an estimate was made of 286 new patients in 1981 . Since only $18 \%$ of all patients survive one year it could be calculated that 51 patients diagnosed before 1981 would be in the community to make a figure of $286+51=$ 337 for the prevalence of oesophageal cancer in the region. The actual figure for inpatients who had been in hospital in 1980 and then again in 1981 was 34 .

If the figure of 444 is reduced by the 42 patients from outside the region and the 34 who had been in hospital in 1980, the resultant number is 368 . Given the present accuracy of recording inpatient activity and the current figures for incidence and survival with oesophageal cancer, there are discrepancies and it is not clear what happens to all the patients in a community with this disease, even though events in hospital are recorded. This estimated figure of 286 is accepted as the true incidence in the region, although it is probably an underestimate. The prevalence can be calculated theoretically but no conclusions can be drawn from the number of patients with sesophageal cancer admitted to the hospitals each year.

\section{SURGERY}

In a previous study I suggested from evidence from published work that $58 \%$ of all patients had an operation, of which about two thirds were radical and one third were palliative. ${ }^{2}$ In this study 73 had surgery-only $25 \%$ of the estimated total of 286 . Nevertheless, the ratio among procedures of two thirds radical to one third palliative in this region is similar. In England and Wales, with 3708 oesophageal cancers in 1978, there were 1000 recorded oesophagogastrectomies, $27 \%$ of the total. ${ }^{6}$ The number of oesophagogastrectomies recorded in this particular study excludes those done for lesions of the stomach and cardia. A preliminary search through the North East Thames region figures for 1981 showed 50 procedures involving an oesophageal anastomosis which could be interpreted as an oesophagogastrectomy done for gastric cancer. Extrapolation of these regional figures to the national figures for England and Wales would suggest that fewer than half of the 1000 oesophagogastrectomies are actually done for gastric cancer. It is therefore extremely difficult to calculate what percentage of patients with oesophageal cancer have surgery. The estimate of $58 \%$ from published work is too high and the figure should be $25 \%$ or less.

The operative mortality in the North East Thames region for radical surgery was $35 \%$ in 1981 and $43 \%$ in 1980 . If both radical and palliative surgery are considered together the postoperative hospital mortality was $33 \%$. If death in hospital after 30 days is excluded the postoperative mortality figure for radical surgery was $21 \%$. From published work the mean figure for radical surgery is $30 \%,{ }^{2}$ with a range from 1 to $83 \%$ but with various definitions for operative mortality. This must confirm that oesophagogastrectomy has the highest mortality of any routinely performed surgical procedure.

There is controversy about how operative mortality data should be presented. Some surgeons exclude palliative surgery and others include only deaths in hospital within 30 days. In this study there were insufficient data on palliative surgery to know whether a bypass procedure, intubation, or nothing was done when radical removal of the tumour was impossible. The operative mortality of radical surgery included all deaths in hospital at any date after surgery provided it was during the same admission. The mean time of death was on the 27 th day. If postoperative radical surgery mortality had been defined as death within 30 days of the operation the underestimate of mortality would have been $42 \%$. Operative mortality should probably not be defined as that occurring within $\mathbf{3 0}$ days of surgery, but should include all postoperative deaths in hospital after surgery whenever they happened.

\section{RADIOTHERAPY}

Radiotherapy was performed in 55 patients; this figure is probably an underestimate. The North East Thames region has nine radiotherapy centres with a linear accelerator or a cobalt source for radiation. If the patient is fit and can swallow radiotherapy may be done on an outpatient basis and would therefore not be included in the inpatient analysis. There are no data on whether this radiotherapy was palliative or radical. Only 55 out of 286 received radiotherapy, $20 \%$ of all the estimated patients. Since this treatment can provide palliation and in some instances survival figures equal to surgery it would appear that radiotherapy is underused, in spite of adequate provision of these facilities in the region.

\section{costs}

Not only are there medical reasons for auditing hospital activity: there are also financial factors. The inpatient care of patients with oesophageal cancer entailed a total of 10355 days in a hospital bed. A bed in a London teaching hospital costs $£ 886$ per week ( $£ 126$ a day) and no acute hospital bed can be obtained for less than $£ 100$ a day. The total cost of inpatient care of oesophageal cancer in the North East Thames region is therefore certainly well over $£ 1$ million per year. 


\section{POSSIBLE FUTURE IMPROVEMENTS}

What improvements can be made in the future?

Investigations-Ideally every patient who comes into hospital with dysphagia should be assessed with radiology, endoscopy, and histology, although oesophageal cancer can be diagnosed by barium swallow alone.

I believe that every general practitioner should be able to request a barium swallow, which can be done as an outpatient procedure before the patient sees a consultant, the degree of urgency being established by the family doctor to avoid delay. In the records only 179 out of 444 patients $(40 \%)$ had an endoscopy after admission to hospital. This may be an underestimate because not all endoscopies are reported, and many of the patients may have been admitted for other reasons with oesophageal cancer as a secondary diagnosis, but could they swallow?

Surgery-There are 136 hospitals in the region; patients with oesophageal cancer were looked after in 52 and surgery was performed in 23 . There are 16 districts and in an ideal world each of these should have only one acute hospital looking after emergencies, presumably with an intensive care unit. It is government policy to have only one casualty department and one acute hospital for each district; should not major surgery also be confined to this one hospital ? Could better results be obtained by centralisation of surgical procedures?

Surgeons-Presumably with over 80 general surgeons and nine thoracic surgeons there are enough surgeons in the region although many would argue about their distribution. Thirty seven of the general surgeons and all the thoracic surgeons performed surgery to oesophageal cancer but only five consultants looked after more than 10 patients a year. No one consultant and no unit would or should look after all the patients in the region. But could more specialisation achieve better results?

Palliation-Most patients are unfit for surgery but it is essential that the dysphagia is relieved somehow so that they can swallow at least liquids to take nutrition and analgesics. In 1981 this type of palliation by endoscopic intubation was not being used frequently enough. Only 112 had an endoscopy and 80 had intubation performed at endoscopy. Only 55 patients had radiotherapy. One hundred and seventy nine patients had no treatment aimed at relief of their dysphagia, and 106 of these died in hospital. Are patients receiving adequate palliation for their dysphagia? Are they being offered it ?

Terminal care-One hundred and nine patients died in hospital without any therapeutic procedure being recorded and 99 were estimated as dying at home. The overall national figures show that one third of all patients with cancer die from causes not directly related to the disease. There is always disagreement whether patients with cancer should die at home or in hospice type accommodation. One thing should be certain and that is that they should not have to die in an acute hospital bed. The role of a surgeon should be to perform radical or palliative operations and dilate or intubate to relieve mechanical obstruction. The fact that most cancers present late and $35 \%$ of the patients are aged 75 or older on presentation excludes major surgery in the majority, but when dysphagia has been relieved or palliated other colleagues can help. Terminal care of a patient who can swallow saliva and liquids is an essential part of the treatment of oesophageal cancer. Terminal care when the patient cannot swallow saliva or liquids is very difficult, and patients should not have to receive intravenous fluids. The London Hospital is very lucky to have St Joseph's Hospice and the Macmillan Service in Tower Hamlets. Are there enough hospices in the region? Why do so many patients die in hospital, what is being done for them, and could they be looked after at home or in a hospice ?

There is much discussion about the lack of resources in the National Health Service. If patients with oesophageal cancer are receiving less than their full share of adequate treatment is the problem to be solved by more cash or merely better organisation by the doctors? A barium swallow and an endoscopy for dysphagia surely take preference over dyspepsia related to excess alcohol intake without requiring more resources. Relief of dysphagia by surgery, radiotherapy, or intubation must be a priority and there is no evidence that there are not enough specialists to do this. Investigation and treatment are not being limited by lack of money. On the other hand, the medical profession appears to be wasting a large amount of money by admitting patients for terminal care into acute hospital beds. Most important of all, would it not be more humane for patients to die at home or in a hospice if they so desired?

I thank Mr Brian Russell, Hospital Activity Analysis officer for the North East Thames Regional Health Authority, for providing the data; Mr Stephen Evans, statistician of the London Hospital Medical College for help with the analysis; and Dr Alan Silman of the London Hospital clinical epidemiological unit for advice.

\section{References}

1 Earlam RJ, Cunha-Melo JR, Donnan SPB, Evans SJW. The epidemiology of oesophageal cancer with special reference to England and Wales. Ital F Gastroenterol 1982;14:244-9.

2 Earlam RJ, Cunha-Melo JR. Oesophageal squamous cell carcinoma: a critical review of surgery. Br f Surg 1980;67:381-90.

${ }^{3}$ Earlam RJ, Cunha-Melo JR. Oesophageal squamous cell carcinoma: a critical review of radiotherapy. Br $\mathcal{J}$ Surg 1980;67:457-61.

4 Office of Population Censuses and Surveys. Cancer statistics. Survival England and Wales, 1971-73. London: HMSO, 1980.

5 Office of Population Censuses and Surveys. Mortality statistics. London: HMSO, 1980. (DH5 no 6.)

${ }^{6}$ Allen-Mersh TG, Earlam RJ. General surgical workload in England and Wales. $\mathrm{Br}$ Med f 1983;287:1115-18.

(Accepted 15 March 1984)
As a help to diagnosing chronic prostatic infection the doctor is recommended to massage the patient's prostate and obtain a prostatic bead for examination. I have never succeeded in doing this. Would not an examination of the whole ejaculate provide a better guide to the diagnosis?

Culture of the seminal fluid obtained by ejaculation has been suggested in those patients in whom prostatic secretions cannot be obtained on massage. ${ }^{1}$ Interpretation may be difficult, however, owing to contamination of the ejaculate by urethral organisms. It is not difficult to diagnose true chronic bacterial prostatitis as it is invariably associated with recurrent bacteriuria. If segmented urine cultures are obtained ${ }^{2}$ the prostate can be proved to be the site of bacterial persistence even between episodes of bacteriuria when the patient is symptomless. Many patients with a vague aching discomfort in the perineum often with radiation to the testicles and discomfort on voiding are labelled as having prostatitis. There is not uncommonly an overlap with sexual difficulties, mainly poorly sustained erections and premature ejaculation. The problem may well be psychosexual or stress mediated and be associated with stress frequency and urgency of micturition. Such patients are not helped by being labelled as having prostatitis and do not produce prostatic secretions on massage or inflammatory cells in their urine voided after a prostatic massage. The term prostatodynia is used to describe this group of patients with no evidence of prostatic inflammation. Non-bacterial prostatitis is more common than true bacterial prostatitis and is of obscure aetiology. These patients without demonstrable bacteriuria do have inflammatory cells in urine voided after prostatic massage, but antimicrobial agents are largely ineffective. ${ }^{3}$-J C GINGELL, consultant urologist and lecturer in urology, Bristol.

Moblcy DF. Semen cultures in the diagnosis of bacterial prostatitis. $\mathcal{f}$ Urol 1975 114:83-5.

Mears FM, Stamey TA. Bacteriologic localisation patterns in bacterial prostatiti

and urethritis. Imestigative Urology 1968;5:492-518.
Stamcy TA. Prostatis. $尹 R$ Soc Med 1981;74:22-40. 